\title{
Promiscuous gene expression in the thymus: a matter of epigenetics, miRNA, and more?
}

\section{Olga Ucar * and Kristin Rattay}

Division of Developmental Immunology, German Cancer Research Center, Heidelberg, Germany

\section{Edited by:}

Geraldo Aleixo Passos, University of São Paulo, Brazil

\section{Reviewed by:}

Mitsuru Matsumoto, University of

Tokushima, Japan

Mireia Guerau-de-Arellano, The Ohio

State University, USA

\section{*Correspondence:}

Olga Ucar, Division of Developmental Immunology, German Cancer

Research Center, Im Neuenheimer

Feld 280, Heidelberg 69126, Germany

e-mail: o.ucar@dkfz.de
The induction of central tolerance in the course of T cell development crucially depends on promiscuous gene expression (pGE) in medullary thymic epithelial cells (mTECs). mTECs express a genome-wide variety of tissue-restricted antigens (TRAs), preventing the escape of autoreactive $T$ cells to the periphery, and the development of severe autoimmunity. Most of our knowledge of how pGE is controlled comes from studies on the autoimmune regulator (Aire). Aire activates the expression of a large subset of TRAs by interacting with the general transcriptional machinery and promoting transcript elongation. However, further factors regulating Aire-independent TRAs must be at play. Recent studies demonstrated that pGE in general and the function of Aire in particular are controlled by epigenetic and post-transcriptional mechanisms. This mini-review summarizes current knowledge of the regulation of pGE by miRNA and epigenetic regulatory mechanisms such as DNA methylation, histone modifications, and chromosomal topology.

Keywords: mTEC, promiscuous gene expression, Aire, epigenetic, miRNA, tolerance, tissue-restricted antigen

\section{INTRODUCTION}

The establishment of central tolerance to all organs of the body is to a large extent mediated by the unique ability of medullary thymic epithelial cells (mTECs) to express a vast variety of self-antigens. This so-called promiscuous gene expression (pGE) encompasses a genome-wide selection of tissue-restricted antigens (TRAs), and so far no involvement of tissue-specific transcription factors in their regulation has been observed in the thymus $(1,2)$. pGE sets the scope of self tolerance, i.e., clonal deletion and Treg induction, and faulty thymic expression of even a single TRA can precipitate organ-specific autoimmunity (3-5); however, we still lack a coherent model incorporating and explaining all the intricacies of pGE.

Most of our knowledge of the molecular control of pGE comes from studies on autoimmune regulator (Aire) $(6,7)$. Mutations in the AIRE gene cause a rare monogenic autoimmune disorder autoimmune polyendocrinopathy-candidiasis-ectodermal dystrophy (APECED), affecting multiple organs with a preference for endocrine glands $(8,9)$. The Aire-deficient mouse model recapitulates the autoimmune phenotype observed in human patients (10).

Autoimmune regulator controls the expression of a subset of TRAs by interacting with the general transcriptional machinery and promoting transcript elongation. Aire does not have a dedicated DNA recognition motif, and it is unclear how it is targeted to an exclusive set of TRA-encoding genes, which is largely conserved across species (11). Depending on the cellular context, Aire can induce the expression of different sets of genes (12), suggesting that the epigenetic landscape of mTECs plays a role in defining Aire targets. Moreover, many TRAs are expressed in TECs in an Aireindependent manner implying that additional factors also regulate pGE. Noteworthy, a set of cell-lineage-specific TFs has proven dispensable for promiscuous transcription of the corresponding target genes in the thymus (13-16). Thus, the likelihood of tissuespecific TFs responsible for the Aire-independent gene regulation in mTECs or TFs acting in concert with Aire remains an open question.

Recent studies documented a role for epigenetic and posttranscriptional mechanisms in regulating pGE in general and the function of Aire in particular (14, 17-19). Indeed, both APECED patients (8) and mouse mutants (20) display variability in the disease severity and the organs affected depending on different genetic backgrounds, indicating that other genetic or epigenetic components define the exact course of the individual disease. Here, we briefly review our current knowledge of how DNA methylation, histone modification, and miRNA may influence pGE and mTEC maintenance.

\section{EPIGENETIC REGULATION OF pGE}

Transcription factor-triggered gene expression is cross-regulated by a number of enzymes, which modify the DNA itself (DNA methylation) or the histones (histone post-translational modifications). Recent studies showed that DNA and histone modifications can alter the promoter structure and accessibility to the extent of adding new TF binding sites, thus shaping the level and pattern of gene expression and, consequently, developmental decisions (21). Accumulating evidence suggests that all these modifications might also be involved in regulating pGE.

\section{DNA METHYLATION}

Methylation of cytosines in $\mathrm{CpG}$ dinucleotides is essential for the regulation of embryonic development, cell lineage progression, gene expression, and chromatin structure (22) and is implicated in several human diseases (23). The majority of CpGs in the human genome are methylated, whereas CpG islands at the transcription start sites of housekeeping genes are hypomethylated 


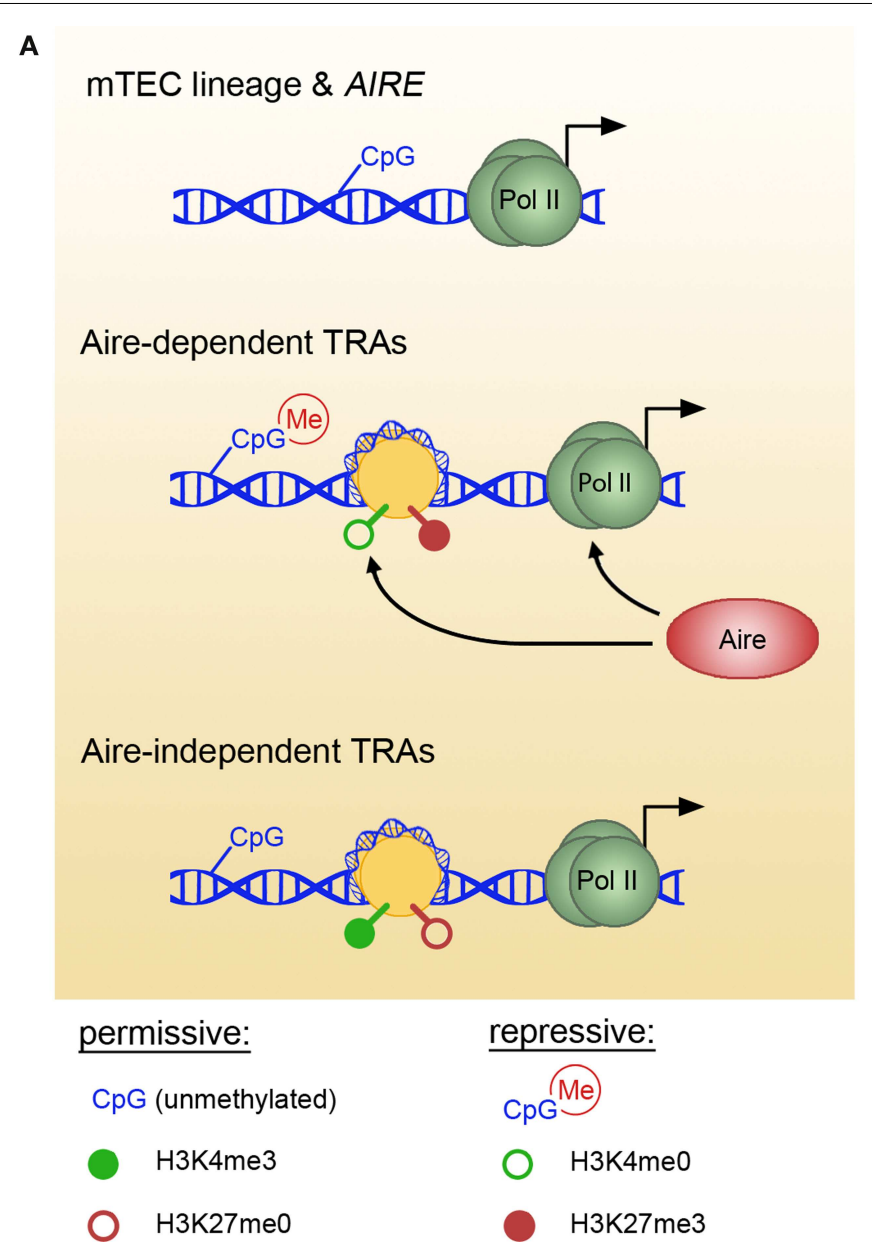

FIGURE 1 | Epigenetic marks in mTECs vary between TRA pools and with mTEC maturation. (A) In mTECs, the loci of TEC lineage-specific genes and various TRA pools bear different epigenetic marks. Thus, lineage genes and AIRE (top) are characterized by low DNA methylation content (permissive), Aire-dependent genes (middle) favor repressive marks, whereas Aire-independent genes (bottom) are maintained in a permissive state. Aire specifically binds $\mathrm{H} 3 \mathrm{~K} 4 \mathrm{me} 0$, facilitating polll recruitment and transcript

\section{mTEC development}

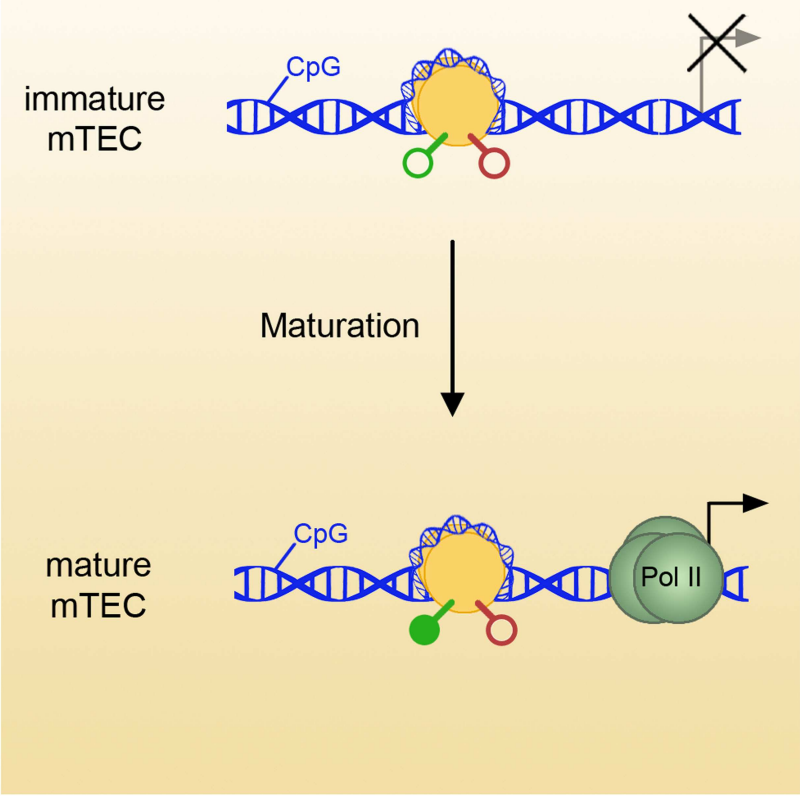

elongation. (B) Aire-independent TRA loci undergo epigenetic changes with mTEC maturation, indicating that permissive marks are acquired in a stepwise fashion and maintained before the onset of gene expression. For example, in immature mTECs, Csn2 genomic locus exists in an epigenetically neutral state, and the appearance of permissive H3K4me3 modification coincides with $\mathrm{mTEC}$ maturation and the onset of transcription. TRAs, tissue-restricted antigens; mTEC, medullary thymic epithelial cell.

(24). As a rule, DNA methylation inversely correlates with gene expression level: a high degree of DNA methylation at promoter regions prevents the binding of transcription factors to their DNA-binding motives and results in transcriptional silencing $(21,22)$. DNA methylation pattern reflects the developmental status of cells with respect to lineage commitment/progression. Thus, the gene loci of myeloid-specific TFs and their binding sites are hypermethylated in the cells of the lymphoid lineage (25). Consistently, demethylation of promoter regions facilitates lineage-specific gene expression, e.g., $\mathrm{CD}^{+} \mathrm{T}$ cell markers are specifically unmethylated and highly expressed in T cells committed to the CD8 lineage (25). Comparison of the methylation patterns of stem/progenitor cells and lineage-committed cells of various tissues demonstrated that the changes in methylation occur at certain lineage-specific gene promoters rather than in extended chromosomal clusters; moreover, this specific methylation pattern is maintained in cells and defines their identity (25-27).

Several recent reports on DNA methylation profiles in mTECs suggest that this epigenetic modification might also pertain to the control of mTEC lineage commitment and pGE; however, the exact specificity and significance of DNA methylation remains unclear (Figure 1A). A number of TEC-specific genes are hypomethylated in mTECs in contrast to other thymic cell types and peripheral tissues (28), suggesting that this pattern arises during lineage commitment and defines mTEC cellular identity. In vitro and ex vivo studies demonstrated that the AIRE gene promoter is hypomethylated in mTECs $(29,30)$, which indicates its transcription-permissive state. In contrast, the Fgg (fibrinogen gamma chain) gene, which encodes a liver-specific protein promiscuously expressed in an Aire-dependent manner, is hypermethylated in both Aire-expressing and Aire-deficient 
mTECs (28). Further intriguing results were obtained from the comparison of the DNA methylation state of the casein locus and the Gad1 (Gad67) promoter between immature and mature adult mTECs (14). The DNA methylation pattern of both regions exists in a permissive state already in immature mTECs, presumably allowing rapid promoter activation after mTEC maturation (Figure 1B). Interestingly, the Csn2 promoter showed progressive demethylation in mature mTECs during embryonic development, which preceded the onset of gene expression (14). Together with the reported hypermethylation of Fgg locus (28), these observations suggest that DNA methylation pattern of TRA loci in mTECs does not reflect the promoter activity in the same way as in other peripheral tissues. In summary, although mTECs seem to adapt the "peripheral" DNA methylation pattern for their lineage-specific genes, they might employ a different strategy for genes expressed promiscuously.

\section{HISTONE MODIFICATION}

Post-translational modifications of histone N-terminal tails are known to be essential in the regulation of transcription, chromatin structure, DNA repair and replication, and alternative splicing. They control gene expression by both recruiting effector proteins, the so-called readers of histone marks, and through changing the compaction state of the chromatin $(21,31)$. Histone modifications fall into active (promoting transcription) and repressive marks and are associated with different chromatin compaction states (32). Active histone marks at promoter and enhancer regions influence polII assembly and elongation through interaction with the basic transcriptional machinery: $\mathrm{H} 4$ acetylation and $\mathrm{H} 3$ trimethylation at K4 are recognized by TFIID and mediate polII assembly at promoters $(33,34)$.

In some instances, active and repressive marks can co-exist within the same region. Thus, embryonic stem cells display overlapping repressive and permissive histone modifications at developmental genes, maintaining their inactivity in steady state, but allowing for rapid activation when differentiation starts (35). Other examples of a poised, bivalent histone code at lineagespecific gene promoters have been observed in $\mathrm{CD}^{+} \mathrm{T}$ cell lineages (36). The coexistence of active (H3K4me3) and repressive (H27K4me3) marks at promoters of lineage-defining transcription factors GATA3, Tbet, Rorc, and Foxp3 endows different $\mathrm{CD}^{+} \mathrm{T}$ cell subtypes with the plasticity to rapidly crossdifferentiate into another subtype in response to environmental stimuli (37).

Studies of histone modifications in mTECs indicate that the histone code plays a role in the regulation of pGE, although the exact mechanisms employed might differ for different sets of TRAs (Figure 1A). Aire-dependent genes in mTECs are characterized by a lack of $\mathrm{H} 3 \mathrm{~K} 4$ trimethylation and enrichment in repressive $\mathrm{H} 3 \mathrm{~K} 27 \mathrm{me} 3(17,38)$. Aire has been shown to specifically bind to unmethylated $\mathrm{H} 3 \mathrm{~K} 4$, targeting genes in a state of low $\mathrm{H} 3 \mathrm{~K} 4 \mathrm{me} 3$ or $\mathrm{H} 3$ acetylation (17). Aire binding was correlated with an increase in $\mathrm{H} 3 \mathrm{~K} 4 \mathrm{me} 3$ and polII recruitment to Aire-dependent gene promoters, implying that Aire facilitates the establishment of active histone marks (17). After polII assembly, Aire assists in the transcriptional elongation through facilitating $\mathrm{p}$-TEFIIb recruitment $(39,40)$. Since $\mathrm{p}$-TEFIIb recruitment requires active histone marks at the enhancer regions (41), involvement of Aire in reading enhancer histone code remains an intriguing possibility.

In contrast to Aire-dependent genes, Aire-independent TRAs seem to favor permissive histone modifications (38). In a recent study, Kyewski and colleagues assessed the chromatin state in mature and immature mTECs at two specific loci encoding Aireindependent TRAs, namely Csn2 and Gad1 (14). Both gene promoters were characterized by permissive histone marks; furthermore, in the case of the Csn 2 promoter, $\mathrm{H} 4$ acetylation and H3K4me3 marks increased with mTEC maturation (Figure 1B). Interestingly, repressive $\mathrm{H} 3 \mathrm{~K} 27 \mathrm{me} 3$ marks were absent from Csn 2 promoter in both immature and mature mTECs, suggesting that at least some TRA promoters maintain a neutral rather than repressed steady state in immature mTECs (14). Whether a similar poising of chromatin occurs in Aire-dependent gene loci early in mTEC lineage progression remains to be determined.

In summary, the histone code of TRA promoters in mTECs can exist in either permissive (Aire-independent genes) or bivalent (Aire-dependent genes) states. One of many functions of Aire seems to be reading and modifying the histone marks, but it is still unclear how their initial deposition is regulated. To this end, functional studies of chromatin modifiers in TECs should shed new light on the mechanisms mTECs employ to achieve and maintain a transcriptionally poised state of TRA-coding genes. It is also pertinent to understand the epigenetic differences between Aire-dependent and -independent genes, which might lead to the identification of factors controlling Aire-independent pGE.

\section{EPIGENETIC LANDSCAPE FLEXIBILITY IN mTECs}

Recent studies revealed that DNA methylation and histone modifications function in a cooperative manner to re-shape chromatin $(21,42)$, and promoter activity can be predicted by the combination of both (43). The stability of transcriptionally active sites largely depends on the cellular and developmental context; more changes in the epigenetic landscape occur during development and lineage progression than in terminally differentiated cells (41). Is this also the case for pGE? On the population level, mTECs express thousands of genes promiscuously, but their global DNA methylation profile is not significantly different from that of peripheral tissues (28). One should, however, consider that individual TRAs are expressed by only a minor fraction of mTECs [1-3\% on average; $(38,44)]$. This might result in an under-representation of TRA-specific epigenetic marks in a population analysis. In this respect, studies of single TRA loci within the mTEC subpopulations expressing that specific TRA will be more informative than global epigenomic approaches. Given that pGE increases in its complexity during mTEC maturation and that even in mature mTECs the expression of individual TRAs seems to be transient (45), the epigenetic landscape in the thymus might turn out to be more flexible and dynamic than in other tissues.

Thus, in the case of the casein locus DNA methylation pattern is established before the histone code and both exist in a permissive state even before Csn2 mRNA can be detected [Ref. (14); Figure 1B]. Since Csn2 seems to be somewhat special with regard to its expression frequency in mTECs (44), the sequential establishment and cooperation of $\mathrm{CpG}$ context and histone marks should be examined in other, less frequent TRA loci at 
different stages of mTEC development. The interplay between histone marks and DNA methylation pattern can result in the organization of actively transcribed loci in transcriptional factories (46), demonstrating the influence of epigenetic marks on genome topology. Recently, emerging evidence suggests that higher order interactions between chromosomal regions in cis and trans might impose further influence on gene expression $(47,48)$. Importantly, stochastic interchromosomal interactions can account for gene expression heterogeneity in a population: for example, colocalization of $\beta$-globin gene and its enhancer was observed in $5-10 \%$ of cells in a population and correlated with a $\sim 100$-fold increase of $\beta$-globin expression in these "jackpot" cells (49). The fact that only a small proportion of mTECs expresses a given TRA at a given time together with a recent observation of TRA loci co-localization (45) suggests that such higher order organization might regulate patterns of gene co-expression in single mTECs in the context of pGE.

\section{POST-TRANSCRIPTIONAL REGULATION OF pGE}

miRNA represents a class of small $(\approx 22 \mathrm{nt})$ RNA molecules involved in the post-transcriptional control of gene expression, acting as switches and fine-tuners of translation (50). Primary miRNA transcripts are polII-dependent and undergo two steps of post-transcriptional processing: by Drosha and DGCR8 in the nucleus and by Dicer and TRBP in the cytoplasm [Ref. (51); Figure 2A]. The mature miRNA are incorporated into the RNAinduced silencing complex (RISC), binding of which to the target mRNA causes a translational block and subsequent mRNA decay $(52,53)$. Target recognition depends on a sequence-specific interaction between the target mRNA $3^{\prime}$ UTR and the seed sequence of miRNA (54). More than half of all protein-coding genes in mammals are regulated by miRNA, and many of them have binding sites for several unrelated miRNA in their $3^{\prime}$ UTR. Additional complexity arises from the fact that a single miRNA can affect the expression of multiple targets. Studies of miRNA function in various tissues revealed complex balanced miRNA-mRNA interaction networks, regulating tissue homeostasis, cell fate decisions, and disease progression (55).

Several recent studies suggest that miRNA may be involved in the regulation of pGE. We showed that a number of miRNA exhibit subset-specific expression in TECs isolated from murine or human thymus; a substantial overlap between miRNA signatures of both species suggests that miRNA expression profiles in TECs are evolutionarily conserved (18). We also demonstrated that maturation-dependent expression of certain miRNA in mTECs correlates with Aire expression (18). Furthermore, changes in miRNA signature have been reported upon Aire knockdown in cell culture (56) and in Aire null mutant thymi (18). Whether Aire directly regulates transcription of miRNA-encoding genes in mTECs and whether such a regulation is a part of stochastic pGE remains to be determined. Since miRNA-encoding genes can use alternative promoters and many miRNA are located in introns $(57,58)$, Aire might be involved in the direct control of miRNA transcription as well as in the miRNA biogenesis coupled to host mRNA processing. Interestingly, Aire has been implicated in Lin28-dependent regulation of let-7 miRNA in ES cells (59). As for Aire being regulated by miRNA, a recent report showed that Aire expression could be controlled by miRNA-220b in an artificial cell culture system (60). It is unclear whether this regulation occurs in human or mouse mTECs in vivo, and no conserved miRNA target sites in Aire mRNA have been predicted in silico by the currently available target prediction tools.

miRNA expression in the thymic epithelium is indispensable for the establishment of central tolerance. Thus, TEC-specific ablation of Dicer or DGCR8 (and therefore all mature miRNA) leads to premature thymic involution, diminished $\mathrm{T}$ cell output and increased susceptibility to autoimmune disease (18, 61-63). The lack of Dicer in TECs leads to a dramatic decline in pGE-a possible underlying cause of the breach in central tolerance. Interestingly, pGE decline affects both Aire-dependent and -independent TRAs in mTECs and cTECs (18), and precedes the loss of TEC cellularity $(18,62)$. The premature involution phenotype of Dicer and DGCR8 mutants is recapitulated in the mouse model lacking miR-29a (61). However, these latter mutants do not exhibit the defects in epithelial organization that result from the loss of all canonical miRNA $(61,63)$ and show only mild delayed impairment of Aire-dependent pGE (18), suggesting that miRNA other than miR-29a play a role in TEC maintenance and function. Further investigation of the mTEC-specific miRNA and their targets will be needed to comprehend the miRNA-dependent regulation of pGE.

How do post-transcriptional inhibitors facilitate pGE? First, in rare cases, miRNA were shown to activate rather than repress gene expression, e.g., through binding to the $5^{\prime}$-UTR [Ref. (64); Figure 2A]. Gene expression activation can also happen indirectly after the miRNA-mediated downregulation of proteins involved in transcriptional repression or RNA decay $(53,65)$. Finally, miRNA could affect pGE indirectly by promoting the maturation of mTECs. Indeed, FoxN1-Cre-mediated loss of Dicer causes alterations in mature mTEC surface antigen profiles (18) and might impair the early stages of mTEC lineage progression (Figure 2B). The fact that mTEC lineage progression and terminal differentiation seem to be unaffected in miR-29a mutants (18) suggests that other miRNA play a role in these processes. Of note, stemness, differentiation, and senescence of keratinocytes seem to be controlled by a complex network of p63 and several miRNA (66). Given the close parallels between keratinocyte and mTEC differentiation (67) further studies on miRNA function in the thymus should reveal whether a similar network determines turnover, maintenance, and function of mTECs.

Apart from being required for mTEC development and pGE, TEC-specific miRNA might play a role in other mechanisms of central tolerance establishment. One of these mechanisms involves a transfer of TRAs from mTECs to dendritic cells (68). Though it is unclear by which precise means the antigens are shared, exosome transfer is a possible route (69). Intriguingly, a recent study showed that human thymic exosomes contain TRAs and TECspecific miRNA (70). miRNA transfer via exosomes was shown to be functionally relevant in various settings. Thus, $\mathrm{T}$ cells share their miRNA by this pathway with antigen-presenting cells and other T cells $(71,72)$. Whether transfer of miRNA from mTECs to dendritic cells indeed takes place via exosomes and the functional significance of this exchange will be clarified in future studies. 


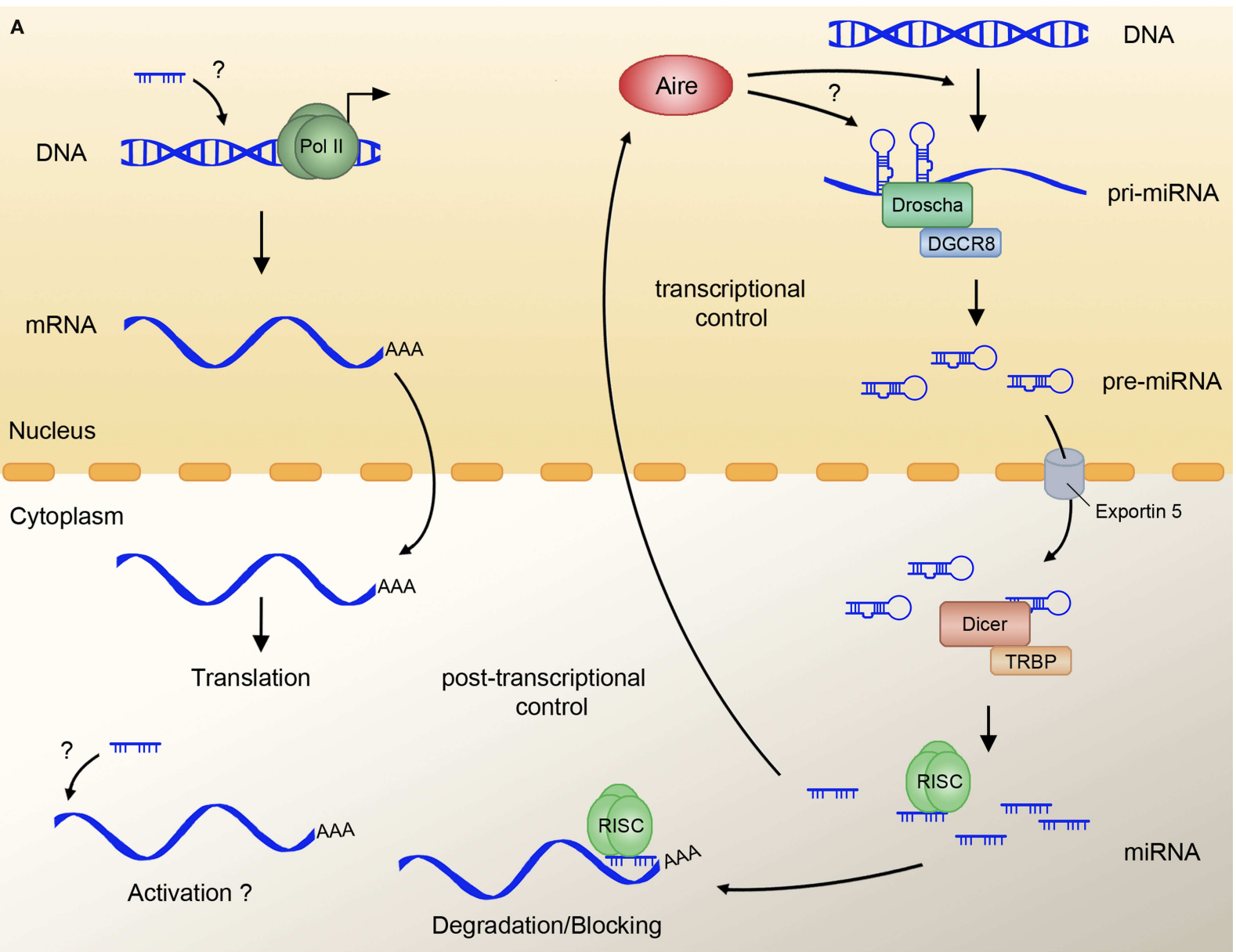

B

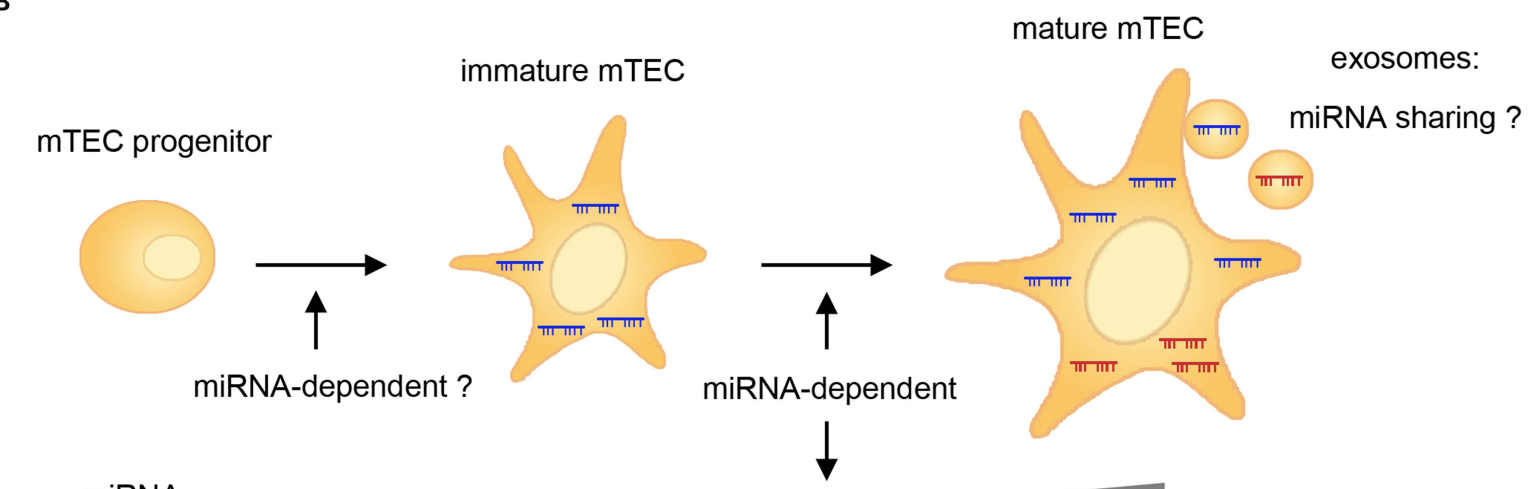

तागाт MiRNA

गानाI mature mTEC-specific miRNA

pGE \& Aire

FIGURE 2 | miRNAs regulate pGE and mTEC maturation. (A) The majority of miRNA primary transcripts (pri-miRNA) are generated by polll and further processed by Drosha/DGCR8 in the nucleus to the stage of pre-miRNA. Pre-miRNA are exported into the cytoplasm by Exportin 5, and further cleaved by the Dicer complex, resulting in mature miRNA. These can be incorporated into the RNA-induced silencing complex (RISC), which binds the target mRNA and usually mediates translational block and/or mRNA degradation. In mTECs, miRNA are indispensable for the expression of Aire and Aire-dependent and -independent TRAs. The mechanism of miRNA action in pGE is not known, and it might involve activation of transcription or translation of TRAs. Aire might be involved in regulating the expression of miRNA-encoding genes and in the generation of miRNA precursors from the so-called miRtrons. (B) mTEC maturation and pGE rely on an intact miRNA pathway, as Dicer deletion in TECs blocks different stages of mTEC lineage progression. Several miRNA are specifically upregulated upon MTEC maturation; however, their exact function and the influence they exert on TRA and Aire expression remains to be determined. The fact that mTEC-specific miRNA are found in human thymic exosomes suggest the possibility of mTECs sharing these small regulators with other antigen-presenting cells in the course of central tolerance induction. RISC, RNA-induced silencing complex; mTEC, medullary thymic epithelial cell; pGE, promiscuous gene expression. 


\section{CONCLUDING REMARIS}

Promiscuous expression of peripheral antigens in the thymus keeps autoimmunity at bay; grasping its exact molecular mechanism will lead to a better understanding of how central tolerance is established and maintained. Transcription factor-mediated gene expression has been shown to go hand in hand with epigenetic and post-transcriptional regulation in many peripheral tissues. Recent studies of these modes of regulation in mTECs suggest that epigenetic marks are deposited and interpreted in an unconventional way in the course of pGE, and that miRNA play an important role in maintaining TRA expression. The future challenge lies in finding out how exactly mTECs utilize ubiquitous epigenetic and post-transcriptional mechanisms to achieve and maintain their extraordinarily broad expression profiles. Will pGE eventually turn out to employ a unique scenario of gene regulatory modes for the sake of preserving tolerance?

\section{ACKNOWLEDGMENTS}

The authors thank Prof. Bruno Kyewski for critical reading of the manuscript and helpful suggestions. OU was supported by the German Cancer Research Center intramural funding, and KR by the European Research Council (ERC-2012-AdG to Bruno Kyewski).

\section{REFERENCES}

1. Derbinski J, Schulte A, Kyewski B, Klein L. Promiscuous gene expression in medullary thymic epithelial cells mirrors the peripheral self. Nat Immunol (2001) 2(11):1032-9. doi:10.1038/ni723

2. Kyewski B, Derbinski J. Self-representation in the thymus: an extended view. Nat Rev Immunol (2004) 4(9):688-98. doi:10.1038/nri1436

3. DeVoss J, Hou Y, Johannes K, Lu W, Liou GI, Rinn J, et al. Spontaneous autoimmunity prevented by thymic expression of a single self-antigen. J Exp Med (2006) 203(12):2727-35. doi:10.1084/jem.20061864

4. Chentoufi AA, Polychronakos C. Insulin expression levels in the thymus modulate insulin-specific autoreactive T-cell tolerance: the mechanism by which the IDDM2 locus may predispose to diabetes. Diabetes (2002) 51(5):1383-90. doi:10.2337/diabetes.51.5.1383

5. Fan Y, Rudert WA, Grupillo M, He J, Sisino G, Trucco M. Thymus-specific deletion of insulin induces autoimmune diabetes. EMBO J (2009) 28(18):2812-24. doi:10.1038/emboj.2009.212

6. Mathis D, Benoist C. Aire. Annu Rev Immunol (2009) 27:287-312. doi:10.1146/ annurev.immunol.25.022106.141532

7. Gray D, Abramson J, Benoist C, Mathis D. Proliferative arrest and rapid turnover of thymic epithelial cells expressing Aire. J Exp Med (2007) 204(11):2521-8. doi:10.1084/jem.20070795

8. Peterson P, Nagamine K, Scott H, Heino M, Kudoh J, Shimizu N, et al. APECED: a monogenic autoimmune disease providing new clues to self-tolerance. Immunol Today (1998) 19(9):384-6. doi:10.1016/S0167-5699(98)01293-6

9. Bjorses P, Aaltonen J, Horelli-Kuitunen N, Yaspo ML, Peltonen L. Gene defect behind APECED: a new clue to autoimmunity. Hum Mol Genet (1998) 7(10):1547-53. doi:10.1093/hmg/7.10.1547

10. Anderson MS, Venanzi ES, Klein L, Chen Z, Berzins SP, Turley SJ, et al. Projection of an immunological self shadow within the thymus by the Aire protein. Science (2002) 298(5597):1395-401. doi:10.1126/science.1075958

11. Danso-Abeam D, Humblet-Baron S, Dooley J, Liston A. Models of Airedependent gene regulation for thymic negative selection. Front Immunol (2011) 2:14. doi:10.3389/fimmu.2011.00014

12. Guerau-de-Arellano M, Mathis D, Benoist C. Transcriptional impact of Aire varies with cell type. Proc Natl Acad Sci U S A (2008) 105(37):14011-6. doi:10.1073/pnas.0806616105

13. Villasenor J, Besse W, Benoist C, Mathis D. Ectopic expression of peripheraltissue antigens in the thymic epithelium: probabilistic, monoallelic, misinitiated. Proc Natl Acad Sci U S A (2008) 105(41):15854-9. doi:10.1073/pnas.0808069105
14. Tykocinski LO, Sinemus A, Rezavandy E, Weiland Y, Baddeley D, Cremer $\mathrm{C}$, et al. Epigenetic regulation of promiscuous gene expression in thymic medullary epithelial cells. Proc Natl Acad Sci U S A (2010) 107(45):19426-31. doi:10.1073/pnas.1009265107

15. Liu Z, Farley A, Chen L, Kirby BJ, Kovacs CS, Blackburn CC, et al. Thymusassociated parathyroid hormone has two cellular origins with distinct endocrine and immunological functions. PLoS Genet (2010) 6(12):e1001251. doi:10.1371/ journal.pgen.1001251

16. Danso-Abeam D, Staats KA, Franckaert D, Van Den Bosch L, Liston A, Gray DH, et al. Aire mediates thymic expression and tolerance of pancreatic antigens via an unconventional transcriptional mechanism. Eur J Immunol (2013) 43(1):75-84. doi:10.1002/eji.201242761

17. Org T, Rebane A, Kisand K, Laan M, Haljasorg U, Andreson R, et al. AIRE activated tissue specific genes have histone modifications associated with inactive chromatin. Hum Mol Genet (2009) 18(24):4699-710. doi:10.1093/hmg/ ddp433

18. Ucar O, Tykocinski LO, Dooley J, Liston A, Kyewski B. An evolutionarily conserved mutual interdependence between Aire and microRNAs in promiscuous gene expression. Eur J Immunol (2013) 43(7):1769-78. doi:10.1002/eji. 201343343

19. Rattay K, Claude J, Rezavandy E, Matt S, Hofmann TG, Kyewski B, et al. Homeodomain-interacting protein kinase 2, a novel autoimmune regulator interaction partner, modulates promiscuous gene expression in medullary thymic epithelial cells. J Immunol (2015) 194(3):921-8. doi:10.4049/jimmunol. 1402694

20. Jiang W, Anderson MS, Bronson R, Mathis D, Benoist C. Modifier loci condition autoimmunity provoked by Aire deficiency. J Exp Med (2005) 202(6):805-15. doi:10.1084/jem.20050693

21. Rothbart SB, Strahl BD. Interpreting the language of histone and DNA modifications. Biochim Biophys Acta (2014) 1839(8):627-43. doi:10.1016/j.bbagrm. 2014.03.001

22. Smith ZD, Meissner A. DNA methylation: roles in mammalian development. Nat Rev Genet (2013) 14(3):204-20. doi:10.1038/nrg3354

23. Robertson KD. DNA methylation and human disease. Nat Rev Genet (2005) 6(8):597-610. doi:10.1038/nrg1655

24. Deaton AM, Bird A. CpG islands and the regulation of transcription. Genes Dev (2011) 25(10):1010-22. doi:10.1101/gad.2037511

25. Bock C, Beerman I, Lien WH, Smith ZD, Gu H, Boyle P, et al. DNA methylation dynamics during in vivo differentiation of blood and skin stem cells. Mol Cell (2012) 47(4):633-47. doi:10.1016/j.molcel.2012.06.019

26. Hemberger M, Dean W, Reik W. Epigenetic dynamics of stem cells and cell lineage commitment: digging Waddington's canal. Nat Rev Mol Cell Biol (2009) 10(8):526-37. doi:10.1038/nrm2727

27. Kaaij LT, van de Wetering M, Fang F, Decato B, Molaro A, van de Werken HJ, et al. DNA methylation dynamics during intestinal stem cell differentiation reveals enhancers driving gene expression in the villus. Genome Biol (2013) 14(5):R50. doi:10.1186/gb-2013-14-5-r50

28. Wu G, Hirabayashi K, Sato S, Akiyama N, Akiyama T, Shiota K, et al. DNA methylation profile of Aire-deficient mouse medullary thymic epithelial cells. BMC Immunol (2012) 13:58. doi:10.1186/1471-2172-13-58

29. Murumagi A, Vahamurto P, Peterson P. Characterization of regulatory elements and methylation pattern of the autoimmune regulator (AIRE) promoter. J Biol Chem (2003) 278(22):19784-90. doi:10.1074/jbc.M210437200

30. Kont V, Murumagi A, Tykocinski LO, Kinkel SA, Webster KE, Kisand K, et al. DNA methylation signatures of the AIRE promoter in thymic epithelial cells, thymomas and normal tissues. Mol Immunol (2011) 49(3):518-26. doi:10.1016/j.molimm.2011.09.022

31. Musselman CA, Lalonde ME, Cote J, Kutateladze TG. Perceiving the epigenetic landscape through histone readers. Nat Struct Mol Biol (2012) 19(12):1218-27. doi: $10.1038 / \mathrm{nsmb} .2436$

32. Li G, Reinberg D. Chromatin higher-order structures and gene regulation. Curr Opin Genet Dev (2011) 21(2):175-86. doi:10.1016/j.gde.2011.01.022

33. Vermeulen M, Mulder KW, Denissov S, Pijnappel WWMP, van Schaik FMA, Varier RA, et al. Selective anchoring of TFIID to nucleosomes by trimethylation of histone H3 lysine 4. Cell (2007) 131(1):58-69. doi:10.1016/j.cell.2007.08.016

34. Moriniere J, Rousseaux S, Steuerwald U, Soler-Lopez M, Curtet S, Vitte AL, et al. Cooperative binding of two acetylation marks on a histone tail by a single bromodomain. Nature (2009) 461(7264):664-8. doi:10.1038/nature08397 
35. Voigt P, Tee WW, Reinberg D. A double take on bivalent promoters. Genes Dev (2013) 27(12):1318-38. doi:10.1101/gad.219626.113

36. Krawczyk CM, Shen H, Pearce EJ. Functional plasticity in memory T helper cell responses. J Immunol (2007) 178(7):4080-8. doi:10.4049/jimmunol.178.7.4080

37. Zhou L, Chong MM, Littman DR. Plasticity of CD4+ T cell lineage differentiation. Immunity (2009) 30(5):646-55. doi:10.1016/j.immuni.2009.05.001

38. Sansom SN, Shikama-Dorn N, Zhanybekova S, Nusspaumer G, Macaulay IC, Deadman ME, et al. Population and single-cell genomics reveal the Aire dependency, relief from Polycomb silencing, and distribution of self-antigen expression in thymic epithelia. Genome Res (2014) 24:1918-31. doi:10.1101/gr. 171645.113

39. Oven I, Brdickova N, Kohoutek J, Vaupotic T, Narat M, Peterlin BM. AIRE recruits $\mathrm{P}-\mathrm{TEFb}$ for transcriptional elongation of target genes in medullary thymic epithelial cells. Mol Cell Biol (2007) 27(24):8815-23. doi:10.1128/MCB. 01085-07

40. Giraud M, Yoshida H, Abramson J, Rahl PB, Young RA, Mathis D, et al. Aire unleashes stalled RNA polymerase to induce ectopic gene expression in thymic epithelial cells. Proc Natl Acad Sci U S A (2012) 109(2):535-40. doi:10.1073/pnas.1119351109

41. Dambacher S, de Almeida GP, Schotta G. Dynamic changes of the epigenetic landscape during cellular differentiation. Epigenomics (2013) 5(6):701-13. doi:10.2217/epi.13.67

42. Bartke T, Vermeulen M, Xhemalce B, Robson SC, Mann M, Kouzarides T. Nucleosome-interacting proteins regulated by DNA and histone methylation. Cell (2010) 143(3):470-84. doi:10.1016/j.cell.2010.10.012

43. Karlic R, Chung HR, Lasserre J, Vlahovicek K, Vingron M. Histone modification levels are predictive for gene expression. Proc Natl Acad Sci U S A (2010) 107(7):2926-31. doi:10.1073/pnas.0909344107

44. Derbinski J, Pinto S, Rosch S, Hexel K, Kyewski B. Promiscuous gene expression patterns in single medullary thymic epithelial cells argue for a stochastic mechanism. Proc Natl Acad Sci U S A (2008) 105(2):657-62. doi:10.1073/pnas. 0707486105

45. Pinto S, Michel C, Schmidt-Glenewinkel H, Harder N, Rohr K, Wild S, et al. Overlapping gene coexpression patterns in human medullary thymic epithelial cells generate self-antigen diversity. Proc Natl Acad Sci U S A (2013) 110(37):E3497-505. doi:10.1073/pnas.1308311110

46. Schoenfelder S, Sexton T, Chakalova L, Cope NF, Horton A, Andrews S, et al. Preferential associations between co-regulated genes reveal a transcriptional interactome in erythroid cells. Nat Genet (2010) 42(1):53-61. doi:10.1038/ng.496

47. Fanucchi S, Shibayama Y, Burd S, Weinberg MS, Mhlanga MM. Chromosomal contact permits transcription between coregulated genes. Cell (2013) 155(3):606-20. doi:10.1016/j.cell.2013.09.051

48. Fanucchi S, Shibayama Y, Mhlanga MM. Are genes switched on when they kiss? Nucleus (2014) 5(2):103-12. doi:10.4161/nucl.28352

49. Noordermeer D, de Wit E, Klous P, van de Werken H, Simonis M, Lopez-Jones $\mathrm{M}$, et al. Variegated gene expression caused by cell-specific long-range DNA interactions. Nat Cell Biol (2011) 13(8):944-51. doi:10.1038/ncb2278

50. Mukherji S, Ebert MS, Zheng GX, Tsang JS, Sharp PA, van Oudenaarden A. MicroRNAs can generate thresholds in target gene expression. Nat Genet (2011) 43(9):854-9. doi:10.1038/ng.905

51. Ha M, Kim VN. Regulation of microRNA biogenesis. Nat Rev Mol Cell Biol (2014) 15(8):509-24. doi:10.1038/nrm3838

52. Guo H, Ingolia NT, Weissman JS, Bartel DP. Mammalian microRNAs predominantly act to decrease target mRNA levels. Nature (2010) 466(7308):835-40. doi:10.1038/nature09267

53. Ameres SL, Zamore PD. Diversifying microRNA sequence and function. Nat Rev Mol Cell Biol (2013) 14(8):475-88. doi:10.1038/nrm3611

54. Bartel DP. MicroRNAs: target recognition and regulatory functions. Cell (2009) 136(2):215-33. doi:10.1016/j.cell.2009.01.002

55. Gurtan AM, Sharp PA. The role of miRNAs in regulating gene expression networks. J Mol Biol (2013) 425(19):3582-600. doi:10.1016/j.jmb.2013.03.007

56. Macedo C, Evangelista AF, Marques MM, Octacilio-Silva S, Donadi EA, Sakamoto-Hojo ET, et al. Autoimmune regulator (Aire) controls the expression of microRNAs in medullary thymic epithelial cells. Immunobiology (2013) 218(4):554-60. doi:10.1016/j.imbio.2012.06.013

57. Ozsolak F, Poling LL, Wang Z, Liu H, Liu XS, Roeder RG, et al. Chromatin structure analyses identify miRNA promoters. Genes Dev (2008) 22(22):3172-83. doi:10.1101/gad.1706508
58. Monteys AM, Spengler RM, Wan J, Tecedor L, Lennox KA, Xing Y, et al. Structure and activity of putative intronic miRNA promoters. RNA (2010) 16(3):495-505. doi:10.1261/rna.1731910

59. Bin G, Jiarong Z, Shihao W, Xiuli S, Cheng X, Liangbiao C, et al. Aire promotes the self-renewal of embryonic stem cells through Lin28. Stem Cells Dev (2012) 21(15):2878-90. doi:10.1089/scd.2012.0097

60. Matsuo T, Noguchi Y, Shindo M, Morita Y, Oda Y, Yoshida E, et al. Regulation of human autoimmune regulator (AIRE) gene translation by miR-220b. Gene (2013) 530(1):19-25. doi:10.1016/j.gene.2013.08.015

61. Papadopoulou AS, Dooley J, Linterman MA, Pierson W, Ucar O, Kyewski $\mathrm{B}$, et al. The thymic epithelial microRNA network elevates the threshold for infection-associated thymic involution via miR-29a mediated suppression of the IFN-alpha receptor. Nat Immunol (2012) 13(2):181-7. doi:10.1038/ni. 2193

62. Zuklys S, Mayer CE, Zhanybekova S, Stefanski HE, Nusspaumer G, Gill J, et al. MicroRNAs control the maintenance of thymic epithelia and their competence for T lineage commitment and thymocyte selection. J Immunol (2012) 189(8):3894-904. doi:10.4049/jimmunol.1200783

63. Khan IS, Taniguchi RT, Fasano KJ, Anderson MS, Jeker LT. Canonical microRNAs in thymic epithelial cells promote central tolerance. Eur J Immunol (2014) 44(5):1313-9. doi:10.1002/eji.201344079

64. Orom UA, Nielsen FC, Lund AH. MicroRNA-10a binds the 5'UTR of ribosomal protein mRNAs and enhances their translation. Mol Cell (2008) 30(4):460-71. doi:10.1016/j.molcel.2008.05.001

65. Bruno IG, Karam R, Huang L, Bhardwaj A, Lou CH, Shum EY, et al. Identification of a microRNA that activates gene expression by repressing nonsense-mediated RNA decay. Mol Cell (2011) 42(4):500-10. doi:10.1016/j.molcel.2011.04.018

66. Candi E, Amelio I, Agostini M, Melino G. MicroRNAs and p63 in epithelial stemness. Cell Death Differ (2015) 22:12-21. doi:10.1038/cdd.2014.113

67. Roberts N, Horsley V. Developing stratified epithelia: lessons from the epidermis and thymus. Wiley Interdiscip Rev Dev Biol (2014) 3(6):389-402. doi:10.1002/wdev.146

68. Perry JS, Lio CW, Kau AL, Nutsch K, Yang Z, Gordon JI, et al. Distinct contributions of Aire and antigen-presenting-cell subsets to the generation of selftolerance in the thymus. Immunity (2014) 41(3):414-26. doi:10.1016/j.immuni. 2014.08.007

69. Kyewski B, Feuerer M. Love is in the Aire: mTECs share their assets. Immunity (2014) 41(3):343-5. doi:10.1016/j.immuni.2014.08.013

70. Skogberg G, Gudmundsdottir J, van der Post S, Sandstrom K, Bruhn S, Benson M, et al. Characterization of human thymic exosomes. PLoS One (2013) 8(7):e67554. doi:10.1371/journal.pone.0067554

71. Mittelbrunn M, Gutierrez-Vazquez C, Villarroya-Beltri C, Gonzalez S, SanchezCabo F, Gonzalez MA, et al. Unidirectional transfer of microRNA-loaded exosomes from T cells to antigen-presenting cells. Nat Commun (2011) 2:282. doi:10.1038/ncomms1285

72. Okoye IS, Coomes SM, Pelly VS, Czieso S, Papayannopoulos V, Tolmachova T, et al. MicroRNA-containing T-regulatory-cell-derived exosomes suppress pathogenic T helper 1 cells. Immunity (2014) 41(1):89-103. doi:10.1016/j.immuni. 2014.05.019

Conflict of Interest Statement: The authors declare that the research was conducted in the absence of any commercial or financial relationships that could be construed as a potential conflict of interest.

Received: 18 December 2014; accepted: 17 February 2015; published online: 03 March 2015.

Citation: Ucar $O$ and Rattay $K$ (2015) Promiscuous gene expression in the thymus: a matter of epigenetics, miRNA, and more? Front. Immunol. 6:93. doi: 10.3389/fimmu.2015.00093

This article was submitted to Immunological Tolerance, a section of the journal Frontiers in Immunology.

Copyright (C) 2015 Ucar and Rattay. This is an open-access article distributed under the terms of the Creative Commons Attribution License (CC BY). The use, distribution or reproduction in other forums is permitted, provided the original author(s) or licensor are credited and that the original publication in this journal is cited, in accordance with accepted academic practice. No use, distribution or reproduction is permitted which does not comply with these terms. 